\title{
Korrespondenzen.
}

\section{Bemerkungen über die Wirkung der Salizylsäure auf das} Gehörorgan.

\section{(Zur Diskussion in der Berliner otologischen Gesellschaft} am 10. November 1903.)

Von Dr. Albert Bla u, Obrenarzt in Görlitz.

$\mathrm{Zu}$ dem Bericht über die Sitzung der Berliner otologischen Gesellschaft vom 10. November 1903 (No. 5 dieser Wochenschrift), halte ich es für notwendig einige Bemerkungen zu machen.

Ich nehme Bezug auf die Aeußerungen des Herrn Dr. Haike in der Diskussion über den Vortrag des Herrn San.-Rat Schwabach.

Bereits am 22. September 1903 maclite ich Mitteilung über mehrere große Untersuchungsreihen von Tieren, welche ich mit Natrium salicylicum vergiftet hatte, und zwar auf der otologischen Sektion der Naturforscherversammlung in Kassel. (Der Bericht darïber ist in der Zeitschrift für Ohrenheilkunde Bd: 45, Heft $t$, enthalten.) Die Resultate dieser Versuche, die sich über eine lange Zeit erstreckten und eine genaue histologische Untersuchung auf Serienschnitten zur Grundlage hatten, ergaben, dall Blutungen nach der von Wittmaak vorgeschlagenen Methode der Tötung der Tiere kurz ante mortem fast nie zu seben waren, dal sie zum Teil auch den Tieren fehlten, welche von selbst eingingen, dab ferner Veränderungen in den Ganglienzellen des Ganglion akusticum vorhanden waren. Die ausführliche Beschreibung dieser Versuche ist, wie damals angekündigt, von mir veröffentlicht und wird in einem der nächsten Hefte des Archivs für Obrenheilkunde erscheinen. Es wird dort auf Grund von eingelienden Studien an nach $\mathbf{N i s s l}$, Held etc. behandelten Schnitten mit Siclerheit festgestellt, daß die Verïnderungen, welche die Salizylsiure im Gehörorgan hervorruft, die Ganglienzellen im Ganglion akusticum und Vestibularganglion betreffen.

Ferner glaube ich, auf die Arbeit Alexanders: „Zur Frage der pathologischen Bedeutung der endolymphatischen Labyrinthblutung * (Archiv für Ohrenheilkunde Bd. 59, 25. August 1903) aufmerksam machen zu müssen, deren in der Diskussion ebenfalls niclit Erwähnung getan wurde. Hier wird bewiesen, dal3 auch endolymphatische Blutungen als Folgeerscheinung der Suffokation, bezw. als agonale Erscheinungen auftreten können. Des weiteren gelangte Alexander zu dem Schlub, ,dab es sich bei der Giftwirkung des Chinins (und ich darf jetzt wohl auch hinzufügen, des Salizyls) auf das Ohrlabyrinth um Blutungen handelt, erscheint nunmehr auch bezüglich der endolymphatischen Blutungen widerlegt ${ }^{\alpha}$.

Zum Schluß noch die kurze Bemerkung, dals meine ausführliche Arbeit, die dem Archiv eingesandt ist, vor dem 30. November 1903 abgeschlossen war und ich von der Diskussion wie vom Yortrage in der otologischen Gesellschaft Berlins erst aus dem Referat in dieser Wochenschrift Kenntnis erhalten habe. 